\title{
Normalization integrals of orthogonal Heun functions
}

\author{
Peter A. Becker \\ Center for Earth Observing and Space Research \\ Institute for Computational Sciences and Informatics, \\ and Department of Physics and Astronomy, \\ George Mason University, Fairfax, VA 22030-4444 \\ (pbecker@gmu.edu)
}

\begin{abstract}
A formula for evaluating the quadratic normalization integrals of orthogonal Heun functions over the real interval $0 \leq x \leq 1$ is derived using a simple limiting procedure based upon the associated differential equation. The resulting expression gives the value of the normalization integral explicitly in terms of the local power-series solutions about $x=0$ and $x=1$ and their derivatives. This provides an extremely efficient alternative to numerical integration for the development of an orthonormal basis using Heun functions, because all of the required information is available as a by-product of the search for the eigenvalues of the differential equation.
\end{abstract}

PACS numbers: 02.30.Gp, 02.30.Hq, 02.70.-c, 02.60.Jh

Byline: Normalization integrals of Heun functions

(Accepted for publication in the Journal of Mathematical Physics) 


\section{INTRODUCTION}

Heun's equation ${ }^{1}$ is the most general Fuchsian equation of second order with four regular singular points, and it is therefore of considerable importance in mathematical physics. Special cases of the Heun equation include the hypergeometric, confluent hypergeometric, Lamé, Bessel, Legendre, and Laguerre equations. As a practical matter, the most important solutions to the Heun equation are those orthogonal functions satisfying prescribed boundary conditions at two adjacent singular points. ${ }^{2}$ The development of an orthonormal basis using these functions is a two-step process. The first step is to search for the eigenvalues, and the second is to evaluate the quadratic normalization integrals of the associated eigenfunctions, which are orthogonal Heun functions. The normalization integrals are usually evaluated numerically, which is not an especially efficient procedure given the nature of the eigenfunctions and the associated series representations. In order to provide a useful alternative to numerical integration, in this paper we derive a new formula for directly evaluating the quadratic normalization integrals of orthogonal Heun functions over the real interval $[0,1]$. The formula obtained (eq. 29) utilizes only information available as a by-product of the search for the eigenvalues, and therefore greatly improves both the efficiency and the accuracy of numerical procedures involving Heun functions.

\section{HEUN FUNCTIONS}

We begin by writing Heun's equation in the standard form first adopted by Erdélyi et al. ${ }^{3}$

$$
\frac{d^{2} y}{d x^{2}}+\left(\frac{\gamma}{x}+\frac{\delta}{x-1}+\frac{\epsilon}{x-a}\right) \frac{d y}{d x}+\frac{\alpha \beta x-\lambda}{x(x-1)(x-a)} y=0,
$$

represented by the Riemann $P$-symbol

$$
P\left\{\begin{array}{ccccc}
0 & 1 & a & \infty & \\
0 & 0 & 0 & \alpha & x \\
1-\gamma & 1-\delta & 1-\epsilon & \beta &
\end{array}\right\}
$$

with regular singular points located at $x=0,1, a, \infty$. The parameter $\lambda$ falls outside the domain of the usual Riemann classification scheme, and is therefore referred to as an acces- 
sory (or auxiliary) parameter. In many applications, $\lambda$ plays the role of an eigenparameter. The five exponent parameters $\alpha, \beta, \gamma, \delta, \epsilon$ are connected via Riemann's relation

$$
\alpha+\beta-\gamma-\delta-\epsilon+1=0,
$$

and therefore only four of them are independent. The total number of free parameters is six, and this number cannot be reduced by any transformation.

Heun ${ }^{1}$ used the method of Frobenius to derive local power-series solutions to (1), generating a three-term recursion relation for the expansion coefficients. Two linearly independent power-series (Frobenius) solutions exist in the neighborhood of any one of the singular points, and, in general, analytic continuation of a single Frobenius solution about one singularity into the neighborhood of a second, adjacent singularity generates a linear combination of the two local Frobenius solutions about the second singularity.

The most important solutions are those that are simultaneously local Frobenius solutions about two adjacent singular points. These are referred to as Heun functions, and often arise when physical boundary conditions are applied to solutions of the differential equation. Taking the parameters $\alpha, \beta, \gamma, \delta, a$ to be constants, the problem of finding a Heun function becomes a singular Sturm-Liouville eigenvalue problem for $\lambda$. However, since no formula is available for performing the analytic continuation between two adjacent singularities in the case of the general Heun equation (in contrast to the subcase of the hypergeometric equation), no general closed-form expression for the eigenvalues $\lambda_{n}$ exists. Heun functions are infinite series in general, although in special cases the series truncates, leaving a Heun polynomial.

There are four classes of Heun functions for a given pair of adjacent singularities $x=s_{0}$ and $x=s_{1}$ that are distinguished by the values of the corresponding exponents $\left(\sigma_{0}, \sigma_{1}\right)$. In this paper, we set $s_{0}=0$ and $s_{1}=1$, and classify the Heun functions according to the usual scheme based upon the values of the associated exponents: class I $(0,0)$; class II $(1-\gamma, 0)$; class III $(0,1-\delta)$; and class IV $(1-\gamma, 1-\delta)$. We focus here on the behavior 
of Heun functions within the real interval $0 \leq x \leq 1$, and we assume throughout that $a \notin[0,1]$.

\section{DETERMINATION OF THE EIGENVALUES}

Let the function $y_{0}(\lambda, x)$ be a local Frobenius solution of (1) in the neighborhood of $x=0$, and let the function $y_{1}(\lambda, x)$ be a local Frobenius solution in the neighborhood of $x=1$. The properties of these solutions, including the recursion relation for the expansion coefficients, have been fully discussed. ${ }^{2}$ Let us suppose that these functions are normalized according to the prescription adopted by Heun, so that

$$
\lim _{x \rightarrow 0} x^{-\sigma_{0}} y_{0}(\lambda, x)=\lim _{x \rightarrow 1}(x-1)^{-\sigma_{1}} y_{1}(\lambda, x)=1
$$

It is known from the basic theory of these solutions that in general $y_{0}$ converges for $|x|<$ $\min (1,|a|)$ and $y_{1}$ converges for $|x-1|<\min (1,|a-1|)$. Hence when $a \notin[0,1]$ as assumed here, there exists a region of mutual convergence, within which both $y_{0}$ and $y_{1}$ converge. The region of mutual convergence is contained within the interval $[0,1]$.

The Wronskian of $y_{0}$ and $y_{1}$ is defined by

$$
W(\lambda, x) \equiv y_{0}(\lambda, x) \frac{\partial y_{1}}{\partial x}(\lambda, x)-y_{1}(\lambda, x) \frac{\partial y_{0}}{\partial x}(\lambda, x)
$$

In order to obtain a Heun function, the Wronskian must vanish, and therefore the eigenvalue equation for $\lambda_{n}$ becomes

$$
W\left(\lambda_{n}, x\right)=0
$$

When this condition is satisfied, $y_{0}\left(\lambda_{n}, x\right)$ and $y_{1}\left(\lambda_{n}, x\right)$ are linearly dependent functions (although not equal in general), and the solution to (1) with $\lambda=\lambda_{n}$ is the Heun function $H_{n}(x)$. We set the normalization of $H_{n}(x)$ by stipulating that $H_{n}(x)=y_{0}\left(\lambda_{n}, x\right)$ in the neighborhood of $x=0$. In the neighborhood of $x=1$, we find that $H_{n}(x)=$ $A\left(\lambda_{n}\right) y_{1}\left(\lambda_{n}, x\right)$, where the value of $A\left(\lambda_{n}\right)$ is determined by requiring that $H_{n}(x)$ be continuous at an arbitrary point within the region of mutual convergence of $y_{0}$ and $y_{1}$. 
We can establish the functional form of the Wronskian by examining the self-adjoint version of the Heun equation,

$$
\mathcal{L} y-\lambda \omega(x) y=0,
$$

where the weight function $\omega(x)$ is defined by

$$
\omega(x) \equiv x^{\gamma-1}(x-1)^{\delta-1}(x-a)^{\epsilon-1},
$$

and the operator $\mathcal{L}$ is defined by

$$
\mathcal{L} y \equiv \frac{d}{d x}\left[x^{\gamma}(x-1)^{\delta}(x-a)^{\epsilon} \frac{d y}{d x}\right]+\alpha \beta x \omega(x) y .
$$

Since $y_{0}$ and $y_{1}$ are each solutions of (1) for the same value of $\lambda$, we may write

$$
y_{0}[\mathcal{L}-\lambda \omega(x)] y_{1}-y_{1}[\mathcal{L}-\lambda \omega(x)] y_{0}=0 .
$$

This yields an equation for the Wronskian;

$$
-\frac{1}{W} \frac{d W}{d x}=\frac{\gamma}{x}+\frac{\delta}{x-1}+\frac{\epsilon}{x-a},
$$

with solution

$$
W(\lambda, x)=D(\lambda) x^{-\gamma}(x-1)^{-\delta}(x-a)^{-\epsilon},
$$

where $D(\lambda)$ is an unknown function. Note that the Wronskian vanishes for $D\left(\lambda_{n}\right)=0$, which is independent of $x$. Hence when we evaluate $W(\lambda, x)$ using $(5)$ in order to calculate the eigenvalues using (6), we are free to pick any convenient value for $x$ that lies within the region of mutual convergence of $y_{0}$ and $y_{1}$.

\section{ORTHOGONALITY RELATIONS}

Let $H_{n}(x)$ and $H_{m}(x)$ be Heun functions of the same class associated with eigenvalues $\lambda_{n}$ and $\lambda_{m}$. Since they are each solutions of (1), we have, by analogy with (10),

$$
H_{n}\left[\mathcal{L}-\lambda_{m} \omega(x)\right] H_{m}-H_{m}\left[\mathcal{L}-\lambda_{n} \omega(x)\right] H_{n}=0 .
$$


After simplifying and integrating over the interval $[0,1]$, we obtain

$$
\left(\lambda_{n}-\lambda_{m}\right) \int_{0}^{1} \omega(x) H_{n}(x) H_{m}(x) d x=\left.p(x)\left(H_{m} \frac{d H_{n}}{d x}-H_{n} \frac{d H_{m}}{d x}\right)\right|_{0} ^{1}
$$

where

$$
p(x) \equiv x^{\gamma}(x-1)^{\delta}(x-a)^{\epsilon} .
$$

Based upon the asymptotic behavior of the local Frobenius solutions $y_{0}$ and $y_{1}$, we find that the right-hand side of (13) vanishes when one of the following sets of class-dependent conditions is satisfied;

$$
\begin{array}{lll}
\text { class I: } & \Re \gamma>0, & \Re \delta>0 \\
\text { class II: } & \Re \gamma<2, & \Re \delta>0 \\
\text { class III: } & \Re \gamma>0, & \Re \delta<2 \\
\text { class IV: } & \Re \gamma<2, & \Re \delta<2 .
\end{array}
$$

We shall refer to (15) as the set of existence conditions for orthogonal Heun functions on

the interval $[0,1]$. When these conditions are met, we obtain the standard orthogonality relation

$$
\left(\lambda_{n}-\lambda_{m}\right) \int_{0}^{1} \omega(x) H_{n}(x) H_{m}(x) d x=0
$$

\section{NORMALIZATION INTEGRALS}

Naturally, the integral in (16) does not vanish when $n=m$, and in this case it is necessary to establish its value in order to develop an orthonormal basis using orthogonal Heun functions. Numerical integration is always available as an option, but this is a very inefficient approach to the problem. Other procedures have been devised, the most interesting being the method developed by Erdélyi, ${ }^{4}$ which is based upon expansions of Heun functions as series of degenerate hypergeometric functions (Jacobi polynomials). Lambe and Ward $^{5}$ developed a technique for evaluating normalization integrals for Heun polynomials, but these results are not applicable to the more general (and much more common) case of Heun functions. In this section we develop a new formula for the explicit evaluation of these integrals. 
We can derive a formula for evaluating the quadratic normalization integral

$$
I_{n} \equiv \int_{0}^{1} \omega(x)\left[H_{n}(x)\right]^{2} d x
$$

by generalizing the approach taken in the preceding section. Proceeding as before, we note that since $y_{0}(\lambda, x)$ and $y_{0}\left(\lambda_{n}, x\right)$ are each Frobenius solutions of (1) in the neighborhood of $x=0$, it follows by analogy with (12) that

$$
y_{0}(\lambda, x)\left[\mathcal{L}-\lambda_{n} \omega(x)\right] y_{0}\left(\lambda_{n}, x\right)-y_{0}\left(\lambda_{n}, x\right)[\mathcal{L}-\lambda \omega(x)] y_{0}(\lambda, x)=0,
$$

where $\lambda_{n}$ is an eigenvalue and $\lambda$ is arbitrary. After simplifying and integrating with respect to $x$, we now obtain

$$
\begin{aligned}
\left(\lambda-\lambda_{n}\right) \int_{0}^{x} \omega & \left(x^{\prime}\right) y_{0}\left(\lambda, x^{\prime}\right) y_{0}\left(\lambda_{n}, x^{\prime}\right) d x^{\prime} \\
& =p(x)\left[y_{0}\left(\lambda_{n}, x\right) \frac{\partial y_{0}}{\partial x}(\lambda, x)-y_{0}(\lambda, x) \frac{\partial y_{0}}{\partial x}\left(\lambda_{n}, x\right)\right],
\end{aligned}
$$

where the right-hand side vanishes as $x \rightarrow 0$ provided the appropriate set of existence conditions in (15) is satisfied.

Upon examination of (19), we find that both sides of the equation vanish as $\lambda \rightarrow \lambda_{n}$. We can therefore establish the value of the indefinite integral in the limit $\lambda \rightarrow \lambda_{n}$ using L'Hôpital's rule, which yields

$$
\begin{aligned}
\int_{0}^{x} \omega\left(x^{\prime}\right)[ & \left.y_{0}\left(\lambda_{n}, x^{\prime}\right)\right]^{2} d x^{\prime} \\
& =p(x)\left[y_{0}\left(\lambda_{n}, x\right) \frac{\partial^{2} y_{0}}{\partial \lambda \partial x}\left(\lambda_{n}, x\right)-\frac{\partial y_{0}}{\partial \lambda}\left(\lambda_{n}, x\right) \frac{\partial y_{0}}{\partial x}\left(\lambda_{n}, x\right)\right] .
\end{aligned}
$$

To obtain a formula for the desired normalization integral $I_{n}$, we must let $x \rightarrow 1$ in (20), and this requires analytic continuation of $y_{0}$ into the neighborhood of $x=1$. For general values of $\lambda$, we write the analytic continuation of $y_{0}$ as

$$
y_{0}(\lambda, x)=A(\lambda) y_{1}(\lambda, x)+B(\lambda) \tilde{y}_{1}(\lambda, x)
$$

where $y_{1}$ is the Frobenius solution about $x=1$ with exponent zero, and $\tilde{y}_{1}$ is the Frobenius solution about $x=1$ with exponent $1-\delta$. At this point we shall restrict our attention to 
Heun functions of classes I or II, so that the exponent at $x=1$ is zero. This restriction will be removed later. Note that $B\left(\lambda_{n}\right)$ must vanish so that we obtain a class I or II Heun function when $\lambda=\lambda_{n}$. Substituting into (20), we obtain after some algebra

$$
\begin{aligned}
I_{n} & \equiv \int_{0}^{1} \omega(x)\left[H_{n}(x)\right]^{2} d x \\
& =\left.\lim _{x \rightarrow 1}\left[A y_{1}\left(A \frac{\partial^{2} y_{1}}{\partial \lambda \partial x}+\frac{d B}{d \lambda} \frac{\partial \tilde{y}_{1}}{\partial x}\right)-A \frac{\partial y_{1}}{\partial x}\left(A \frac{\partial y_{1}}{\partial \lambda}+\frac{d B}{d \lambda} \tilde{y}_{1}\right)\right] p\right|_{\lambda=\lambda_{n}},
\end{aligned}
$$

where we have used the fact that in the neighborhood of $x=1, H_{n}(x)$ is the analytic continuation of $y_{0}\left(\lambda_{n}, x\right)$, and in the neighborhood of $x=0, H_{n}(x)$ is identical to $y_{0}\left(\lambda_{n}, x\right)$.

Based upon asymptotic analysis of $y_{1}$ and $\tilde{y}_{1}$, we find that in the limit $x \rightarrow 1$, all of the terms on the right-hand side of (22) vanish except the second one, so that we are left with

$$
I_{n}=\lim _{x \rightarrow 1} p(x) A\left(\lambda_{n}\right) \frac{d B}{d \lambda}\left(\lambda_{n}\right) \frac{\partial \tilde{y}_{1}}{\partial x}\left(\lambda_{n}, x\right) y_{1}\left(\lambda_{n}, x\right) .
$$

Hence we need only evaluate $\frac{d B}{d \lambda}\left(\lambda_{n}\right)$ and $A\left(\lambda_{n}\right)$ in terms of known functions in order to obtain our final result for the normalization integral $I_{n}$. Evaluation of $A\left(\lambda_{n}\right)$ is a simple matter, since the continuity of $H_{n}(x)$ requires that

$$
A\left(\lambda_{n}\right)=\frac{y_{0}\left(\lambda_{n}, x\right)}{y_{1}\left(\lambda_{n}, x\right)}
$$

for any $x$ within the region of mutual convergence of $y_{0}$ and $y_{1}$. Evaluation of $\frac{d B}{d \lambda}\left(\lambda_{n}\right)$ is slightly more complicated. We begin by differentiating (21) with respect to $x$ to obtain

$$
\frac{\partial y_{0}}{\partial x}(\lambda, x)=A(\lambda) \frac{\partial y_{1}}{\partial x}(\lambda, x)+B(\lambda) \frac{\partial \tilde{y}_{1}}{\partial x}(\lambda, x) .
$$

Solving (21) and (25) for $B(\lambda)$ yields

$$
B(\lambda)=\frac{y_{0} \frac{\partial y_{1}}{\partial x}-y_{1} \frac{\partial y_{0}}{\partial x}}{\tilde{y}_{1} \frac{\partial y_{1}}{\partial x}-y_{1} \frac{\partial \tilde{y}_{1}}{\partial x}} .
$$

Bearing in mind that $B\left(\lambda_{n}\right)=0$, we find upon differentiating (26) with respect to $\lambda$ that

$$
\frac{d B}{d \lambda}\left(\lambda_{n}\right)=\left.\frac{\frac{\partial W}{\partial \lambda}}{\tilde{y}_{1} \frac{\partial y_{1}}{\partial x}-y_{1} \frac{\partial \tilde{y}_{1}}{\partial x}}\right|_{\lambda=\lambda_{n}},
$$


where the Wronskian $W(\lambda, x)$ is defined by (5). We can use (24) and (27) respectively to replace $A\left(\lambda_{n}\right)$ and $\frac{d B}{d \lambda}\left(\lambda_{n}\right)$ in $(23)$, yielding

$$
I_{n}=\left.\lim _{x \rightarrow 1} p \frac{y_{0}}{y_{1}} \frac{\frac{\partial W}{\partial \lambda}}{\tilde{y}_{1} \frac{\partial y_{1}}{\partial x}-y_{1} \frac{\partial \tilde{y}_{1}}{\partial x}} \frac{\partial \tilde{y}_{1}}{\partial x} y_{1}\right|_{\lambda=\lambda_{n}} .
$$

The most singular term in the denominator is the one containing $\frac{\partial \tilde{y}_{1}}{\partial x}$, and therefore in the limit $x \rightarrow 1$ our final result for the normalization integral becomes

$$
\int_{0}^{1} \omega(x)\left[H_{n}(x)\right]^{2} d x=-p(x) \frac{\partial W}{\partial \lambda}\left(\lambda_{n}, x\right) \frac{y_{0}\left(\lambda_{n}, x\right)}{y_{1}\left(\lambda_{n}, x\right)}
$$

In passing to this expression, we have made use of the fact that $\frac{\partial W}{\partial \lambda}\left(\lambda_{n}, x\right) p(x)$ and $y_{0}\left(\lambda_{n}, x\right) / y_{1}\left(\lambda_{n}, x\right)$ are both independent of $x$. Hence the right-hand side of (29) is actually an invariant, which may be evaluated at any point within the region of mutual convergence of the local power-series solutions $y_{0}$ and $y_{1}$.

\section{EXTENSION TO HEUN FUNCTIONS OF CLASSES III AND IV}

We have derived our main result (29) under the assumption that $H_{n}(x)$ is a class I or II Heun function. In the case of a Heun function of class III or IV, the exponent at $x=1$ is $1-\delta$, and the derivation presented above must be modified slightly. First we note that (20) remains valid because it is written in terms of the local solution $y_{0}$ about $x=0$. The analytic continuation of $y_{0}$ into the neighborhood of $x=1$ is still performed using (21), except now we interchange the definitions of $y_{1}$ and $\tilde{y}_{1}$, so that $y_{1}$ is the local Frobenius solution about $x=1$ with exponent $1-\delta$ and $\tilde{y}_{1}$ is the local solution about $x=1$ with exponent zero. In this case we again require $B\left(\lambda_{n}\right)=0$, and following the same procedure as before we regain expression (22). Due to the fact that the definitions of $y_{1}$ and $\tilde{y}_{1}$ have been interchanged, in the limit $x \rightarrow 1$, we find by asymptotic analysis that only the final term in (22) now contributes, so that in this case we obtain

$$
I_{n}=\lim _{x \rightarrow 1}-p(x) A\left(\lambda_{n}\right) \frac{d B}{d \lambda}\left(\lambda_{n}\right) \frac{\partial y_{1}}{\partial x}\left(\lambda_{n}, x\right) \tilde{y}_{1}\left(\lambda_{n}, x\right)
$$


Evaluation of $A\left(\lambda_{n}\right)$ and $\frac{d B}{d \lambda}\left(\lambda_{n}\right)$ proceeds exactly as before, and we simply regain expressions (24) and (27). Using these results in (30) now yields

$$
I_{n}=\lim _{x \rightarrow 1}-\left.p \frac{y_{0}}{y_{1}} \frac{\frac{\partial W}{\partial \lambda}}{\tilde{y}_{1} \frac{\partial y_{1}}{\partial x}-y_{1} \frac{\partial \tilde{y}_{1}}{\partial x}} \frac{\partial y_{1}}{\partial x} \tilde{y}_{1}\right|_{\lambda=\lambda_{n}} .
$$

This is similar to (28), except now the most singular term in the denominator is the one containing $\frac{\partial y_{1}}{\partial x}$, and therefore in the limit $x \rightarrow 1$ we obtain

$$
I_{n}=-p(x) \frac{\partial W}{\partial \lambda}\left(\lambda_{n}, x\right) \frac{y_{0}\left(\lambda_{n}, x\right)}{y_{1}\left(\lambda_{n}, x\right)}
$$

which is identical to (29). We are therefore led to the following general conclusion: Equation (29) holds for Heun functions of any class, provided the existence conditions (15) are satisfied. We discuss the significance of this result and its natural role in computational algorithms below.

\section{COMPUTATIONAL IMPLICATIONS}

Equation (29) has important practical consequences for the design of numerical algorithms used to develop orthonormal systems based on Heun functions, which are the solutions of interest in many mathematical and physical situations. In such cases the first step towards solution is the search for the associated eigenvalues $\lambda_{n}$. This search must proceed numerically in general, due to the lack of a closed-form expression for $\lambda_{n}$ in terms of the parameters $\alpha, \beta, \gamma, \delta, a$. The eigenvalues are obtained by isolating the roots of the Wronskian in (6). The root finding usually proceeds via Newton's method or possibly some more sophisticated algorithm. Most of these techniques require the evaluation of $W$ and $\frac{\partial W}{\partial \lambda}$ in order to generate a revised estimate of the true root $\lambda_{n}$, and the evaluation of these functions in turn involves the determination of the quantities

$$
y_{0}, \frac{\partial y_{0}}{\partial x}, \frac{\partial y_{0}}{\partial \lambda}, \frac{\partial^{2} y_{0}}{\partial \lambda \partial x}, y_{1}, \frac{\partial y_{1}}{\partial x}, \frac{\partial y_{1}}{\partial \lambda}, \frac{\partial^{2} y_{1}}{\partial \lambda \partial x}
$$

at each iteration. The values of $y_{0}$ and $y_{1}$ can be obtained using the well-known powerseries representations, and the values of the derivatives can be obtained using term-by-term 
differentiation. It is straightforward to demonstrate that the radii of convergence of the series for the derivatives are identical to those for the corresponding fundamental series, which are discussed in $\S$ II.

Once the eigenvalues $\lambda_{n}$ have been determined to acceptable precision, one generally needs to evaluate the associated quadratic normalization integrals $I_{n}$ in order to develop a set of orthonormal basis functions $h_{n}(x)$ using

$$
h_{n}(x) \equiv \frac{H_{n}(x)}{I_{n}^{1 / 2}},
$$

with normalization

$$
\int_{0}^{1} \omega(x)\left[h_{n}(x)\right]^{2} d x=1 .
$$

The conventional approach to the problem of evaluating $I_{n}$ is to integrate (17) numerically, in which case thousands of evaluations of $H_{n}(x)$ would generally be required in order to establish the value of $I_{n}$ to reasonable accuracy. However, such an inefficient procedure is no longer necessary with the availability of (29), because it allows the determination of $I_{n}$ to high precision using only the values of $y_{0}, y_{1}$, and $\frac{\partial W}{\partial \lambda}$ obtained in the final iteration of the root-finding stage of the algorithm. Hence no substantial additional calculation is necessary in order to determine $I_{n}$. The computational time required for developing orthonormal systems of Heun functions can therefore be reduced by several orders of magnitude by using (29) instead of numerical integration.

We close by making a comparison between the method for evaluating $I_{n}$ outlined here and that suggested by Erdélyi, ${ }^{4}$ which utilizes Svartholm's ${ }^{6}$ expansions of Heun functions as series of degenerate hypergeometric functions, essentially Jacobi polynomials. First of all, it is worth noting that in Erdélyi's method, the root-finding approach is the same as that outlined above, because his procedure assumes prior knowledge of the eigenvalues. With the eigenvalues already determined, the calculation of the coefficients for the Jacobi expansion proceeds via a three-term recursion relation similar to that derived by Heun for the coefficients of the power-series expansion. Using the familiar result for the quadratic 
normalization integrals of the Jacobi polynomials, it is a simple matter to determine $I_{n}$ from the coefficients of the Jacobi expansion. While Erdélyi's method is interesting from the point of view of functional analysis, it is obvious that his procedure entails much more computation than the evaluation of $I_{n}$ using (29), which we again emphasize requires nothing more than information available as a by-product of the search for the eigenvalues. In conclusion, we point out that the application of L'Hôpital's rule used here to evaluate the normalization integrals of Heun functions can also be used to obtain similar results for more general Sturm-Liouville problems.

\section{REFERENCES}

${ }^{1} \mathrm{~K}$. Heun, Zer Theorie der Riemann'schen Functionen zweiter Ordnung mit vier Verzweigungspunkten, Math. Ann., 33, pp. 161-179 (1889).

${ }^{2}$ A. Ronveaux, ed., Heun's Differential Equations (Oxford University Press, New York, NY, 1995).

${ }^{3}$ A. Erdélyi, W. Magnus, F. Oberhettinger, and F. G. Tricomi, Higher Transcendental Functions, vol. III (McGraw-Hill, New York, NY, 1955).

${ }^{4}$ A. Erdélyi, Certain expansions of solutions of the Heun equation, Quart. J. Math. Oxford Ser., 15, pp. 62-69 (1944).

${ }^{5}$ C. G. Lambe and D. R. Ward, Some differential equations and associated integral equations, Quart. J. Math. Oxford Ser., 5, pp. 81-97 (1934).

${ }^{6}$ N. Svartholm, Die Lösung der Fuchsschen Differentialgleichung zweiter Ordnung durch hypergeometrische Polynome, Math. Ann., 116, pp. 413-421 (1939). 